\title{
Direct detection of coupled proton and electron transfers in human manganese superoxide dismutase
}

\author{
J Azadmanesh ${ }^{1}$, W Lutz $^{2}$, L Coates ${ }^{3}$, K Weiss $^{3}$, G Borgstahl ${ }^{4}$ \\ ${ }^{1}$ Univ. Nebraska Medical Center, Omaha, NE, ${ }^{2}$ University of Nebraska Medical Center, Omaha, NE, \\ ${ }^{3}$ Oak Ridge National Laboratory, Oak Ridge, TN, ${ }^{4}$ The Eppley Inst For Cancer Res. \\ jahaun.azadmanesh@unmc.edu
}

Human manganese superoxide dismutase (MnSOD) is a critical oxidoreductase found in the mitochondria matrix. Concerted proton and electron transfers (CPETs) are used by the enzyme to rid the mitochondria of O2•-, a precursor to other reactive oxygen species (ROS) that are harmful in excessive amounts. The mechanism of CPETutilizing enzymes are difficult to discern due to the inability to directly detect the protonation states of specific residues and solvent molecules involved in catalysis while controlling the redox state of the enzyme. Here, we utilize neutron diffraction of redox-controlled MnSOD crystals to yield all-atom structures of Mn3+SOD and $\mathrm{Mn2+SOD}$ revealing five sites of differential protonations. A novel mechanism is introduced from the direct observation of a glutamine amide-imidic acid tautomerization and three locations of low-barrier hydrogen bonds that change with the oxidation state of the metal. Quantum calculations provide insight into electronic modulation and thermodynamic properties of the observed structures.

Acta Cryst. (2020). A76, a134 